\title{
IV. Zeitschriften und Veröffentlichungen von Gesellschaften
}

1. Astronomische Abhandlungen. Ergänzungshefte zu den Astronomischen Nachrichten. Akademie-Verlag, Berlin. - Band 12 Nr. 2.

2. Abhandlungen (Utschonye Sapiski) der Shdanow-Staatsuniversität des Leninordens in Leningrad. Serie der mathematischen Wissenschaften. Leningrad. - Nr. 22 (Astronomie) = Nr. 136 der ganzen Reihe = Publ. Astr. Obs. Leningrad Tom 15.

3. Acta Astronomica. Krakau. - AA(a) Vol.5 S. 19-50, AA(c) Vol. 4 S. $113-$ 128.

4. Annalen der Physik. Johann Ambrosius Barth, Leipzig. - 6. Folge Band 7 $=$ Band 442 der ganzen Reihe.

5. Annales d'Astrophysique. - Tome 13 Nr. 1-4.

6. Annales de Physique. Masson et Cie, Editeurs, Paris. - Sér. 12 Tome 5 Nr. 1-12.

7. Annales Françaises de Chronométrie. Besançon. - Année 20 Trimestre $1-4$.

8. Anzeiger. Osterreichische Akademie der Wissenschaften. Mathematischnaturwissenschaftliche Klasse. Wien. - 87. Jahrgang Nr. 1-15.

9. Arkiv för Astronomi. Utgivet av K. Svenska Vetenskapsakademien Stockholm. - Band 1 Häfte 2.

10. L'Astronomie. Revue Mensuelle d'Astronomie, de Météorologie et de Physique du Globe. Bulletin de la Société Astronomique de France. Paris. 64 année, Jan.-Déc. 1950.

11*. Berichte (Doklady) der Akademie der Wissenschaften der UdSSR. MoskauLeningrad. - Tom 70-75, jeweils Heft 1-6.

12*. Berichte (Doklady) der Akademie der Wissenschaften der armenischen Sowjetrepublik. Erewan. - Tom 11 Nr. 4. Tom 12 Nr.4.

13. Physikalische Berich te. Herausgegeben im Auftrag der Physikalischen Gesellschaft Württemberg.Baden-Pfalz. Akademie-Verlag, Stuttgart und Berlin. - Band 29 Heft 1-12.

14. Physikalische Blätter. Physik Verlag, Mosbach (Baden). - 6. Jahrgang Heft 1-12.

15*. Bote (Westnik) der Akademie der Wissenschaften der UdSSR. MoskauLeningrad. - Tom 20 Nr. 3, 11.

16*. Bote (Westnik) der Moskauer Universität. - Tom 5 Nr. 8.

17. Bulletin Astronomique, Publié par l'Observatoire de Paris. - Tome 15 Fasc. 2-4.

18. Bulletin de l'Association Française d'Observateurs d'Etoiles Variables. Lyon. - Tome 9, vgl. AJB 49 Ref. 11218. Tome 10 Fasc. 1, 2.

19. Bulletin der Astronomisch-Geodätischen Gesellschaft der UdSSR. Verlag der Akademie der Wissenschaften der UdSSR, Moskau-Ieningrad. Nr. 8 (15), Nr. 9 (16). 
20. Bulletin horaire du Bureau International de l'Heure. Observatoire de Paris. -2me Série: Janvier-Décembre 1950. Série D: Janvier-Août 1950.

21. Bulletin of the Astronomical Institutes of Czechoslovakia. National Observatory Praha. - Vol. 2 Nr. 1-5.

22. Bulletin of the Astronomical Institutes of the Netherlands. Haarlem. Vol. 11 Nr. 408-418.

23. Ciel et Terre. Bulletin mensuel de la Société Belge d'Astronomie, die Météorologie et de Physique du Globe. Uccle-Brüssel. - Année 66 Nr. 1-12.

24. British Astronomical Association Circular. London. - Nr. 313-320.

25. The Japan Astronomical Study Association Circular (japanisch-nur teilweise referiert). - Nr. 75-92.

26. Coelu m. Periodico Bimestrale per la Divulgazione dell'Astronomia. Bologna. - Vol. 18 Nr. 1-12.

27. Comptes Rendus hebdomadaires des Séances de l'Académie des Sciences. Paris. - Tome 230-231.

28. Documentation des Observateurs. Institut d'Astrophysique de Paris. 1950 Nr. $1-12$.

29. Endeavour. Eine Vierteljahres-Übersicht von Fortschritten der Wissenschaften im Dienste der Menschheit. London. - Band 9 Nr. 33-36.

30. Ergebnisse der exakten Naturwissenschaften. Springer-Verlag, GöttingenBerlin-Heidelberg. - Band 23.

31. Extraits d'Astronomie et de Géophysique du Centre National de la Recherche Scientifique. (Continuation de la Bibliographie Mensuelle de l'Astronomie) Paris. - Vol. 11 Nr. 1-12.

32. Forschungen und Fortschritte. Nachrichtenblatt der Deutschen Wissenschaft und Technik. Akademie-Verlag, Berlin. - Jabrgang 26 Heft 1-24.

33. Gazette astronomique. Bulletin de la Société d'Astronomie d'Anvers. Année 32 Nr. 1-12. Gesamtnummer 361-372.

34. Glasnik. Societas Scientiarum Naturalium Croatica. Periodicum Mathematico-Physicum et Astronomicum. Zagreb. - Ser. (2) T. 5 Nr. 1-5.

35. Heelal. Tijdschrift van de Vereniging voor Sterrenkunde, Meteorologie, Geophysica en aanverwante Wetenschappen. Antwerpen. - Jaargang 6 Aft. 1 -5.

36. Hemel en Dampkring. J. B. Wolters, Groningen. - Jaargang 48 Afl. 1-12 (von A. Güttler referiert).

37*. Isis. An International Review devoted to the History of Science and Civilization. - Vol. 41 (wird nur auszugsweise referiert).

38. Astronomisches Journal. Akademie der Wissenschaften der UdSSR. MoskauLeningrad. - Tom 27 Nr. $1-6$.

39. The Astronomical Journal. Yale Univ. Office, New Haven. - Vol. 55 Nr. 2-6. Gesamtnummer 1182-1186.

40. The Astrophysical Journal. Chicago. - Vol. 111 Nr. 1-3. Vol. 112 Nr. 1-3.

41. The Irish Astronomical Journal. Armagh. - Vol. 1 Nr. 1-4.

42. Le Journal de Physique et Le Radium. Publication de la Société Française de Physique. Paris. - Tome 11 Nr. $1-12$. 
43. Journal des Observateurs. Publié avec le concours du Centre National de la Recherche Scientifique. Marseille. - Vol. 33 Nr. 1-12.

44. Journal of Atmospheric and Terrestrial Physics. Butterworth-Springer Ltd., London. - Vol. 1 Nr. 1-2.

45. Journal of Calendar Reform. Published by the World Calendar Association, Inc. New York. - Vol. 20 Quarter 1-4.

46. Journal of Geophysical Research. The continuation of Terrestrial Magnetism and Atmospheric Electricity. The Johns Hopkins Press, Baltimore. - Vol. 55 Nr. $1-4$.

47. Journal of the British Astronomical Association. Oxford. - Vol. 60 Nr. 28. Vol. 61 Nr. 1.

48. Journal of the British Interplanetary Society. London. - Vol. 9 Nr. 1-6 und Annual Report 1950.

49. Journal of the Optical Society of America. Published for the Optical Society of America by American Institute of Physics. - Vol. 40 Nr. 1-12.

50. Journal of the Royal Astronomical Society of Canada. Toronto. - Vol. 44 Nr. 1-6. Gesamtnummer 382-387.

51. Astronomical News Letter. Issued and distributed by the Harvard College Observatory, Cambridge/Mass., for the Committee for the Distribution of Astronomical Literature of the American Astronomical Society. Nr. 47-53.

52. Memoirs of the British Astronomical Association. Oxford. - Vol. 36 Part 3.

53. Memorie della Società Astronomica Italiana. Nuova Serie. Pavia. - Vol. 21 Nr. 1-4.

54*. Meteoritika. Sammlung von Aufsätzen unter Redaktion von W. G. Fessenkow. Verlag der Akademie der Wissenschaften der UdSSR. MoskauLeningrad. - Nr. 7 (keine Unterlagen eingegangen), Nr. 8.

55. Mitteilungen der Astronomischen Gesellschaft. - 1950.

56. Astronomische Nachrichten. Im Auftrage der Deutschen Akademie der Wissenschaften zu Berlin. - Band 278 Heft 3-6, Band 279 Heft 1-2.

57. Na chrichten der Akademie der Wissenschaften in Göttingen. MathematischPhysikalische Klasse, Mathematisch-Physikalisch-Chemische Abteilung. Jahrgang 1950 Nr. 1-7.

58*. Nachrichten (Iswestija) der Akademie der Wissenschaften der UdSSR. Geographische und geophysikalische Serie. Moskau-Leningrad. - Tom 14 Nr. 1, 4.

59*. Nachrichten (Iswestija) der Akademie der Wissenschaften der UdSSR. Physikalische Serie. Moskau-Leningrad. - Tom 14 Nr. 1, 3, 6.

60*. Nachrichten (Iswestija) der Akademie der Wissenschaften der kasachischen Sowjetrepublik. Alma-Ata. - Nr. 90, astrobotanische Serie Heft 1-2.

61*. Nachrichten (Iswestija) der turkmenischen Abteilung der Akademie der Wissenschaften der UdSSR. - Nr. 2, 4.

62. Nachrichtenblatt der Astronomischen Zentralstelle. Voriäufige Mitteilung. Astronomisches Rechen-Institut Heidelberg. - Nr. 82-100.

63. Nachrichtenblatt der Astronomischen Zentralstelle. Astronomisches Rechen-Institut, Heidelberg. - Jahrgang 4 Nr. I-11. 
64. Astronomischer Nachrichtendienst der Archenhold-Sternwarte zu BerlinTreptow. - 2. Jahrgang Nr. 1-12. (Wird nur auszugsweise referiert.)

65. Name-List of Variable Stars. International Astronomical Union. Commission for Variable Stars of the Astronomical Council of the Academy of Sciences of the USSR and the Sternberg Astronomical Institute, Moscow. - Nr. 46.

66. Nature. A Weekly Journal of Science. Macmillan and Co., London; The Macmillan Company, New York. - Vol.165 Nr.4184-4208. Vol. 166 Nr. $4209-4235$.

67. Die Naturwissenschaften. Springer-Verlag, Berlin - Göttingen - Heidelberg. - 37. Jahrgang Heft 1-24.

68. Monthly Notes of the Astronomical Society of South Africa. Cape Town. Vol. g Nr. 1-11.

69. Monthly Notices of the Royal Astronomical Society. London. - Vol. 110 Nr. 1-6.

70. Monthly Notices of the Royal Astronomical Society. Geophysical Supplement. London. - Vol. 6 Nr. 1-2.

71. The Observatory. Oxford. - Vol. 70 Nr. 854-859.

72. Optik. Zeitschrift für das gesamte Gebiet der Licht- und Elektronenoptik. Stuttgart. - Band 6 Heft 1-6, Band 7 Heft $1-6$.

73. Orion. Zeitschrift der Schweizerischen Astronomischen Gesellschaft. Bulletin de la Société Astronomique de Suissé. Genève bzw. Schaffhausen. Band 3 Nr. 26-29.

74. Physica. Den Haag-Amsterdam. - Vol. 16 Nr. 1-12.

75. Popular Astronomy. A Review of Astronomy and Allied Sciences. Northfield, Minn., Carleton College. - Vol. 58 Nr. 1-10.

76*. Priroda (Natur). Moskau-Leningrad. - Tom 39 Nr. 1-12.

77. Proceedings of the National Academy of Sciences of the United States of America. Washington. - Vol. 36 Nr. 1-12.

78. The Proceedings of the Physical Society. Section A, Section B. London. Vol. 63 Nr. $361-372$.

79. Proceedings of the Royal Society. Series A. Mathematical and Physical Sciences. London. - Vol. 201-204 Nr. 1061-1077.

80*. Proceedings of the Section of Science. Koninklijke Akademie van Wetenschappen te Amsterdam.

81*. Publikationen (Trudy) des Geophysikalischen Instituts der Akademie der Wissenschaften der UdSSR. Verlag der Akademie dor Wissenschaften der UdSSR, Moskau-Leningrad. - Nr. 12 (139).

32*. Publikationen (Trudy) des zentralen Forschungsinstituts für Geodäsie, Luftbildaufnahme und Kartographie. Verlag für geodätische und kartographische Literatur, Moskau. - Nr. 70, 73.

83. Publications of the Astronomical Society of the Pacific. San Francisco. Vol. 62 Nr. $364-369$.

84. Publications of the Astronomical Society of Japan. Tokyo. - Vol. 1 Nr. 4. Vol. 2 Nr. 1-2. 
85. Reviews of Modern Physics. Published for the American Physical Society by the American Institute of Physics. Lancaster and New York. Vol. 22 Nr. 1-4.

86. Mathematical Reviews. Published by the American Mathematical Society, Lancaster, Penn. - Vol. 11. Nr. 1-11.

87. The Physical Review. Series 2. Published for the American Physical Society by the American Institute of Physics. Lancaster, Pa., and New York. - Vol. 77 Nr. 1-12. Vol. 78 Nr. 1-12. Vol. 79 Nr. 1-12. Vol. 80 Nr. 1-12.

88. The Review of Scientific Instruments. New Series. Lancaster, New York. - Vol. 21 Nr. 1-12.

89*. Revista Astronomica. Organo Trimestral de la Asociacion Argentina "Amigos de la Astronomia». Buenos Aires.

90. Revue d'Optique Théorique et Instrumentale. Paris. - Tome 29 Nr. 5, $8-9,10$ (die übrigen Nummern sind nicht eingegangen).

91. Rí̌s hvězd. Herausgegeben von der Tschechischen Astronomischen Gesellschaft in Prag. - Band 31 Nr. 1-10 (tschechisch, Inhalt auch in russischer und englischer Sprache) von J. Bouška, Prag, referiert.

92. Naturwissenschaftliche $R$ undschau. Wissenschaftliche Verlagsgesellschaft m.b. H., Stuttgart. - Jahrgang 3 Heft 1-12.

93. Scientia. Internationale Zeitschrift für wissenschaftliche Synthese. Bologna. - Vol. $85 \mathrm{Nr} .1-12$.

94. Sitzungsberichte der Deutschen Akademie der Wissenschaften zu Berlin. Mathematiseh-naturwissenschaftliche Klasse. Akademie-Verlag, Berlin. Jahrgang 1950 Nr. 1.

95. Sitzungsberichte der mathematisch-naturwissenschaftlichen Klasse der Bayerischen Akademie der Wissenschaften zu München. - Jahrgang 1949, 1950.

96. Sitzungsberichte. Osterreichische Akademie der Wissenschaften. Mathematisch-naturwissenschaftliche Klasse. Abteilung IIa, Wien. — Band 158 Heft 1-10. Band 159 Heft 1-10.

97. Sky and Telescope. Harvard College Observatory, Cambridge, Mass. Vol. 9 Nr. 3-12. Vol. 10 Nr. 1-2.

98. Southern Stars. The Journal of the Royal Astronomical Society of New Zealand. - Vol. 14 Nr. 7-10, Serial Nr. 101-104.

99. Die Sterne. Monatsschrift über alle Gebiete der Himmelskunde. Verlag Johann Ambrosius Barth, Leipzig. - 26. Jahrgang Heft 1-12.

100. Sternenwelt. Populäre Zeitschrift für Astronomie und ihre Geschichte. München. Verlag S. Lux, Murnau, Obb., später Sternenwelt-Verlag, München 8. - 2. Jahrgang Heft 1-12.

101. The Strolling Astronomer. Association of Lunar and Planetary Observers. Albuquerque, New Mexico. - Vol. 4 Nr. 1-12.

102. Populär Astronomisk Tidskrjft. Stockholm. - Argång 31 Häfte 1-4.

103. Nordisk Astronomisk Tidsskrift. Kopenhagen. - $1950 \mathrm{Nr} .1-4$.

104. Transactions of the International Astronomical Union. - Vol. 7.

105*. Die Umschau. Halbmonatsschrift für die Fortschritte in Wissenschaft und Technik. Frankfurt am Main. - Wird nur auszugsweise referiert. 
106*. Universitas. Zeitschrift für Wissenschaft, Kunst und Literatur. Stuttgart. - Wird nur auszugsweise referiert.

107*. El Universo. Organo de la Sociedad Astronómico de México. - Jahrgang 1949 und 1950 nicht eingegangen. (1950 von A. Güttler referiert).

108. Urani a. Populært astronomisk Tidsskrift. Kopenhagen. - Band 7 Nr. 1-12.

109. Urania. Revista de Astronomia y Ciencias Afines. Organo Oficial de la Sociedad Astronómica de España y América y de la Unión Nacional de Astronomia y Ciencias Afines. Barcelona. - Ãno 35 Nr. 221-224.

110. Veränderliche Sterne. Bulletin, herausgegeben vom Astronomischen Rat der Akademie der Wissenschaften der UdSSR. Moskau-Leningrad. Tom 7 Nr. 4-6 (70-72).

111. Vierteljahrsschrift der Naturforschenden Gesellschaft Zürich. Verlag Gebr. Fretz AG., Zürich. - Jahrgang 95 Heft 1-4.

112. Weltraumfahrt. Beiträge zur Weltraumforschung und Astronautik. Umschau-Verlag, Frankfurt am Main. - Jahrgang 1950 Heft 1-6.

113. Zeitschrift für Astrophysik. Springer-Verlag, Berlin-Göttingen-Heidelberg. - Band 27 Heft 1-3. Band 28 Heft 1.

114. Zeitschrift für Naturforschung. Verlag der Zeitschrift für Naturforschung, Tübingen. - Band 5 a Heft 1-12.

115. Zeitschrift für Physik. Springer-Verlag, Berlin-Göttingen-Heidelberg. Band 127 Heft 1-12. Band 128 Heft 1-12.

116. Deutsche Hydrographische Zeitschrift. Deutsches Hydrographisches Institut, Hamburg. - Band 3 Heft 1-6.

117. Zentralblatt für Mathematik und ihre Grenzgebiete. Springer-Verlag, Berlin-Göttingen-Heidelberg. - Band 33 Heft 1-10. Band 34 Heft $1-10$. Band 35 Heft 1-10.

118. Astronomisches Zirkular. Herausgegeben vom Büro der Astronomischen Mitteilungen der Akademie der Wissenschaften der UdSSR. Kasan. Nr. 94-108. 